\title{
Evaluación de métodos para la eliminación de la capa adherente de los huevos del botete diana Sphoeroides annulatus (Pisces: Tetraodontidae)
}

\author{
Evaluation of methods for eliminating egg stickiness of the bullseye puffer fish \\ Sphoeroides annulatus (Pisces: Tetraodontidae)
L. Estela Rodríguez-Ibarra1, M. Isabel Abdo-de la Parra1, Gustavo A. Rodríguez-Montes de Oca ${ }^{2}$, M. Selene Moreno-Hernández ${ }^{2}$, Gabriela Velasco-Blanco ${ }^{1}$, Noemí García-Aguilar ${ }^{1}$ y Luis S. Álvarez-Lajonchère ${ }^{1}$ \\ ${ }^{1}$ Centro de Investigación en Alimentación y Desarrollo, A.C., Av. Sábalo-Cerritos S/N, C.P. 82010 Mazatlán, Sinaloa, México. \\ ${ }^{2}$ Facultad de Ciencias del Mar, Universidad Autónoma de Sinaloa. Paseo Claussen S/N, \\ C. P. 82000 Mazatlán Sinaloa, México \\ eibarra@ciad.mx
}

\begin{abstract}
Six different hatching treatments and one control were used to eliminate egg stickiness of fertilized bullseye puffer fish Sphoeroides annulatus eggs in this experiment. Each treatment was carried out in triplicate and the eggs were incubated in fiberglass tanks. A fertilization rate of $96.2 \% \pm$ $2.4 \%$ was obtained and significant differences were observed
\end{abstract}

\section{Introducción}

Los estudios realizados en diferentes disciplinas sobre el cultivo del botete diana Sphoeroides annulatus (Jenyns, 1842) en la unidad Mazatlán del Centro de Investigación en Alimentación y Desarrollo (CIAD), han demostrado que esta especie es altamente resistente al manejo y se reproduce fácilmente en condiciones de cautiverio (Abdode la Parra \& Duncan 2002, Martínez-Palacios et al. 2002, Komar et al. 2004). La carne de especies de la familia Tetraodontidae es de excelente gusto por su sabor y firmeza (Bussing 1995, Martínez-Palacios et al. 2002, Chávez-Sánchez et al. 2008), y en países asiáticos, especies como Takifugu rubripes tienen una gran demanda y un alto precio en el mercado (Kikuchi et al. 2006). El botete diana ha sido consumido desde hace más de tres décadas en localidades del Noroeste de México y se comercializa desde US $\$ 7 \mathrm{~kg}^{-1}$ el organismo entero y hasta US $\$ 11 \mathrm{~kg}^{-1}$ el filete (Chávez-Sánchez et al. 2008, García-Ortega 2009). Los huevos viables del botete diana son esféricos y transparentes, su vitelo contiene un aglomerado de glóbulos de aceite y cuando están fertilizados poseen una capa adhesiva translúcida (Sikkel 1990, Fujita \& Honma 1991), la cual le sirve para adherirse a determinados sustratos para su desarrollo embrionario (Abdo-de la Parra \& Duncan 2002, Komar et al. 2004). En especies como la carpa y el esturión que son cultivadas con éxito en el mundo, se han desarrollado between treatments. The highest hatching rate occurred at 96.4 $\pm 2 \%$ with the protease enzyme solution whilst the lowest rate was observed in the control $(18.8 \pm 5.3 \%)$. The hatching success of the bullseye puffer fish is significantly improved by eliminating egg adhesion.

Key words: Degumming, incubation, hatching, bullseye puffer técnicas para el mejoramiento de gametos de alta calidad biológica, lo cual garantiza la obtención de una gran cantidad de larvas. Los huevos de estas especies, al igual que el botete diana poseen una capa adhesiva y se ha observado que bajo condiciones de cultivo al incubarse se adhieren unos con otros formando masas sólidas, lo cual no permite una buena oxigenación entre los huevos, provocando altas mortalidades a causa de la anoxia y el desarrollo de agentes patógenos como hongos y bacterias (Kowtal et al. 1986, Demska-Zakés et al. 2005). La optimización de la reproducción de la carpa y el esturión y para la obtención de una gran cantidad de larvas, depende en parte de los métodos que utilizan para remover la capa adherente de los huevos. Al inicio del cultivo del botete diana en el CIAD, los huevos se colocaban en placas de vidrio para su incubación obteniendo un porcentaje máximo de eclosión de $80 \%$, en donde se observó el desarrollo de bacterias y hongos (Abdo-de la Parra \& Duncan 2002). El objetivo de este trabajo fue realizar ensayos de diferentes métodos de eliminación de la capa adherente (desgomado) de los huevos del botete diana para incrementar el porcentaje de eclosión.

\section{Material y métodos}

Los huevos fueron obtenidos de una hembra de $874 \mathrm{~g}$ de peso y $33 \mathrm{~cm}$ de longitud total, inducida al desove con la hormona liberadora de gonadotropina análoga (GnRHa) 
en implantes EVAc de liberación rápida preparados en laboratorio $^{1}$ (Zohar et al. 1990). Para la fertilización se utilizó un macho de 795 g y $30 \mathrm{~cm}$ de longitud total, al cual no fue necesario implantarle la GnRHa. La fertilización se realizó artificialmente mezclando los gametos con agua marina filtrada por UV. Se utilizaron 5 g de huevos fertilizados por tratamiento y cada uno se realizó por triplicado, incluido el control.

Los tratamientos consistieron en: a) Solución de enzima proteolítica proteasa (Sigma-Aldrich $\left.{ }^{\circledR}\right)($ alcalasa de acuerdo a Merck $\left.{ }^{\circledR}\right)$ a una concentración de $5 \mathrm{ml} \mathrm{L}^{-1}$ durante $8 \mathrm{~min}$; b) Solución de enzima proteolítica quimotripsina (Sigma-Aldrich ${ }^{\circledR}$ ) a una concentración de $0,024 \mathrm{ml} \mathrm{L}^{-1}$ durante $8 \mathrm{~min}$, posteriormente se eliminó esta solución y se repitió la operación y se agregó nuevamente una concentración de $0,24 \mathrm{ml} \mathrm{L}^{-1}$ de la enzima durante 16 min; c) Solución de ácido tánico (SigmaAldrich $^{\circledR}$ ) en una concentración de $0,5 \mathrm{~g} \mathrm{~L}^{-1}$ durante 4 min; d) Jugo de piña natural $10 \mathrm{ml} \mathrm{L}^{-1}$ durante $3 \mathrm{~min}$, posteriormente se eliminó esta solución y se agregó jugo de piña concentrado durante $3 \mathrm{~min}$; e) Técnica de Woynarovich modificada, se agregó una solución para fertilizar compuesta por cloruro de sodio $\left(4 \mathrm{~g} \mathrm{~L}^{-1}\right)$ y urea (3 $\left.\mathrm{g} \mathrm{L}^{-1}\right)$ disueltas en agua de mar; posteriormente se eliminó y se agregó a los huevos fertilizados una solución de cloruro de sodio $\left(0,4 \mathrm{~g} \mathrm{~L}^{-1}\right)$ y urea $\left(0,3 \mathrm{~g} \mathrm{~L}^{-1}\right)$ disueltas en agua de mar durante $45 \mathrm{~min}$. Finalmente se enjuagaron los huevos con una solución de ácido tánico $\left(0,5 \mathrm{~g} \mathrm{~L}^{-1}\right)$ durante 4 min; f) Solución de leche de vaca $\left(100 \mathrm{ml} \mathrm{L}^{-1}\right)$ la cual se deja actuar en los huevos fertilizados durante 50 min con recambios cada $4 \mathrm{~min}$, posteriormente se agregó arcilla (100 $\left.\mathrm{g} \mathrm{L}^{-1}\right)$ durante $10 \mathrm{~min}$. Para el control se utilizaron huevos fertilizados sin tratamiento los cuales se sembraron sobre placas de vidrio.

Al final de cada tratamiento, los huevos se enjuagaron con agua de mar filtrada con UV y se incubaron en tanques negros circulares de fibra de vidrio de $600 \mathrm{~L}$ de capacidad a una densidad de 33 huevos $\mathrm{L}^{-1}$. La incubación se llevó a cabo con agua de mar previamente filtrada hasta $1 \mu \mathrm{m}$ de retención absoluta y pasada por una lámpara UV, con aireación fuerte (2-5 L min $\left.{ }^{-1}\right)$, manteniendo el oxígeno disuelto en $6 \mathrm{ppm}$ y la temperatura a $25,2^{\circ} \mathrm{C}$ y sin flujo continuo de agua.

Se evaluó el porcentaje de eclosión de los huevos tomando cuatro muestras aleatorias en distintos puntos del tanque con un vaso de precipitado de $250 \mathrm{~mL}$ y se contaron las larvas y los huevos presentes en un litro (Álvarez-Lajonchère \& Hernández-Molejón 2001). Los

${ }^{1}$ University of Maryland Biotechnology Institute, Center of Marine Biotechnology Baltimore, Maryland, Estados Unidos porcentajes de eclosión se calcularon de acuerdo a la siguiente fórmula:

$$
\mathrm{E}=[\mathrm{L} /(\mathrm{L}+\mathrm{H})] \cdot 100
$$

donde $\mathrm{E}$ = porcentaje de eclosión, $\mathrm{L}$ = número de larvas, y $\mathrm{H}=$ número de huevos.

Se determinó la longitud total de las larvas mediante un vernier digital (precisión 0,001 mm).

Los resultados del porcentaje de eclosión fueron transformados a arcoseno y se determinó la normalidad de su distribución mediante la prueba de Bartlett, así como la homocedasticidad de la varianza (prueba de Levene). Los datos presentaron distribución normal y fueron evaluados mediante un análisis de varianza de una vía (ANOVA, STATGRAPHICS Plus 5.1 (Statgraphics $^{\mathrm{TM}}$ net) $(P<0,05)$, y cuando se presentaron diferencias significativas, se analizaron mediante pruebas de comparaciones múltiples de medias de Tukey $(\alpha<0,05)$.

\section{Resultados y discusión}

El porcentaje de fertilización promedio fue de 96,2 \pm $2,4 \%$ con un rango de 92,14 a 98,57\% (Tabla 1). Todos los métodos utilizados eliminaron la capa adherente de los huevos, excepto la enzima quimotripsina. El porcentaje más alto de eclosión se obtuvo con la enzima proteasa $(96,47 \pm 2,0 \%)$, mientras que el más bajo se presentó en los huevos sin tratamiento (control) (18,82 \pm $5,3)$. Los resultados fueron significativamente diferentes $(P=0,0001)$ (Tabla 1$)$. Se ha demostrado que el tiempo de incubación depende de la temperatura (MartínezRodríguez 2003 en Abdo-de la Parra \& Duncan 2002) y en el presente estudio la temperatura promedio de incubación de $S$. annulatus fue de $25,2^{\circ} \mathrm{C}$, lo cual pudo afectar el desarrollo embrionario de los huevos del control y por consiguiente un bajo porcentaje de eclosión; a diferencia de otros estudios donde a temperaturas superiores a $27^{\circ} \mathrm{C}$ obtuvieron mejores porcentajes de eclosión usando huevos con capa adherente (MartínezPalacios et al. 2002, Martínez-Rodríguez 2003).

El empleo de la enzima proteasa resultó ser el método más eficiente, considerando que además de obtener el mayor porcentaje de eclosión, es una técnica sencilla y rápida para desgomar los huevos de botete. Esta enzima es usada muy frecuentemente en criaderos de Europa con gran éxito para desgomar huevos de peces dulceacuícolas como ciprínidos (Cyprinus carpio, Tinca tinca, Barbus lutes) y silúridos (Silurus glanis), como parte de la optimización de la reproducción artificial de dichas especies y con el objetivo de aumentar el porcentaje de eclosión (Al Hazzaa \& Hussein 2003, Gela et al. 2003, Linhart et al. 2000, 2003a, 2003b, 2004, 2006). Otra de 


\section{Tabla 1}

Promedio \pm desviación estándar de fertilización, eclosión y longitud total de las larvas de S. annulatus. Valores con superíndices indican diferencias significativas $(P<0,05)$

Average \pm standard deviation of fertilization rates, hatching rates and total length $S$. annulatus larvae. Different superscripts indicate significant differences $(P<0.05)$

\begin{tabular}{lccc}
\hline Tratamiento & Fertilización (\%) & Eclosión (\%) & Longitud total (mm) \\
\hline Enzima proteolítica proteasa & $98,57 \pm 1,2$ & $96,47 \pm 2,03^{\mathrm{c}}$ & $1,93 \pm 0,17$ \\
Acido tánico & $97,32 \pm 2,3$ & $87,05 \pm 5,39^{\mathrm{bc}}$ & $2,01 \pm 0,10$ \\
Jugo de piña & $98,20 \pm 1,8$ & $89,11 \pm 6,23^{\mathrm{bc}}$ & $1,95 \pm 0,15$ \\
Técnica de Woynarovich & $96,56 \pm 3,6$ & $77,64 \pm 3,52^{\mathrm{b}}$ & $1,90 \pm 0,12$ \\
Leche mas arcilla & $92,14 \pm 3,5$ & $78,08 \pm 0,62^{\mathrm{b}}$ & $1,91 \pm 0,16$ \\
Control & $94,78 \pm 2,2$ & $18,82 \pm 5,39^{\mathrm{a}}$ & $1,92 \pm 0,04$ \\
\hline
\end{tabular}

las técnicas eficientes para desgomar los huevos de $S$. annulatus y con altos resultados de eclosión (89,11 \pm $6,2 \%$ ), fue el jugo de piña, que tiene la ventaja de ser un técnica rápida, sencilla y económica y esta fruta se puede conseguir fácilmente durante todo el año. Thai \& Ngo (2004) usaron jugo de piña para desgomar huevos de carpa y obtuvieron un porcentaje de eclosión de 86,6\%, muy cercano al reportado en el presente estudio.

Con la técnica de ácido tánico se obtuvo también un alto porcentaje de eclosión (87,05 $\pm 5,3 \%)$, y comparado con Demska-Zakés et al. (2005) y Carral et al. (2006), que aplicaron este tratamiento a los huevos de la lucioperca (Sander lucioperca) y de la tenca (Tinca tinca), estos autores reportaron resultados inferiores al presente estudio, de 35 a 52 y 47,3\%, respectivamente. El tiempo de acción del ácido tánico reportado por Carral et al. (2006) de 15 s y Demska-Zakés et al. (2005) de 0,5, 2 y 5 min, lo relacionan directamente a la efectividad de eliminar la capa adherente y mencionan que a menor tiempo no se elimina la adherencia de los huevos los cuales se pegan entre sí o a las paredes de la incubadora. Sin embargo, es recomendable tener precaución con este ácido ya que es tóxico y puede dañar al embrión (Woynarovich 1983, Thai \& Ngo 2004). Demska-Zakés et al. (2005), observaron que, aunque a altas concentraciones el ácido tánico remueve la capa adherente, las larvas al eclosionar no se liberan del corion ya que éste se endurece y muchas de ellas mueren. Este efecto no se observó en los huevos de S. annulatus.

La técnica de Woynarovich modificada (RodríguezGutiérrez \& Garza-Mouriño 1985, Aydin \& Köprücü 2005) es otra de las técnicas empleadas para desgomar huevos de peces dulceacuícolas, particularmente en carpa
(Appelbaum \& Riehl 1997, Köprücü \& Aydin 2004, Aydin \& Köprücü 2005). Los resultados de eclosión obtenidos con esta técnica fueron de 77,64 \pm 3,5\% y con la técnica de leche más arcilla fue de 78,08 $\pm 0,6$. Estos valores no fueron diferentes de los datos de eclosión de S. annulatus en temporadas pasadas (80\%, Abdo-de la Parra \& Duncan 2002), y por lo tanto no revisten un aporte significativo a la mejora de la manipulación de los huevos. Al igual que los trabajos de Kowtal et al. (1986), Al Hazzaa \& Hussein (2003) y Carral et al. (2006), reportan porcentajes de eclosión de huevos de esturiones y ciprínidos menores al $60 \%$ usando la técnica de Woynarovich, la cual ocupa mayor tiempo y con resultados de eclosión bajos. Por otro lado, Linhart et al. (2000, 2003a), compararon la técnica de leche mas arcilla, la cual se utiliza desde la década de los 70, con la técnica de la enzima proteasa en los huevos de la tenca (Tinca tinca), reportando que la enzima es una técnica más eficiente, porque requiere menor tiempo para desgomar a los huevos y se obtiene un porcentaje mayor al método de leche mas arcilla, resultados muy semejantes al presente estudio.

El único tratamiento que no desgomó los huevos de S. annulatus, fue el de la enzima quimotripsina. Linhart et al. (2003b) utilizaron y recomendaron esta enzima y la alcalasa por su efectividad para eliminar la capa adherente de los huevos de la carpa. Por lo tanto, se recomienda que en trabajos futuros se modifique esta técnica en cuanto a dosis y tiempo de exposición para evaluar su efectividad en el desgomado y porcentaje de eclosión de los huevos de $S$. annulatus.

El tiempo de eclosión de las larvas de $S$. annulatus se presentó a las 60 horas post-fertilización (hpf) en todos 


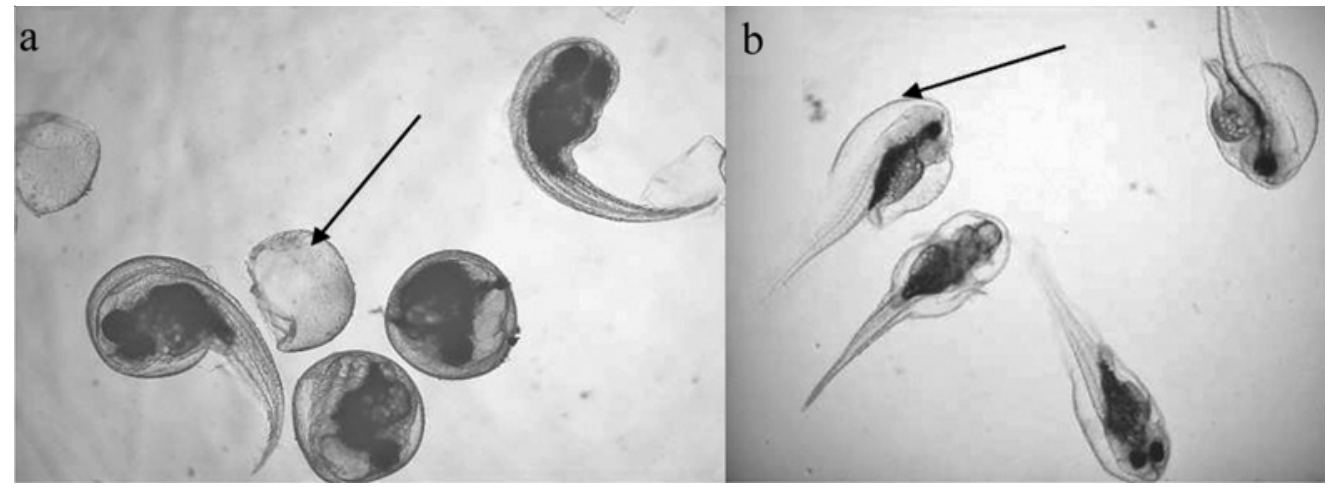

Figura 1

a) Eclosión de las larvas de $S$. annulatuS a) del control y b) del tratamiento con la enzima proteasa. Las flechas indican los residuos del corion

a) Newly hatched $S$. annulatus larvae a) from the control and those b) treated with the protease enzyme. Arrows indicate chorion residues

los tratamientos a excepción del control (84 hpf) y el tratamiento con la enzima proteolítica quimotripsina. La eliminación de la capa adherente parece estar relacionada con el adelanto de la eclosión larval, probablemente debido a las diferencias en grosor del corion (Fig. 1). Las larvas recién eclosionadas midieron 1,93 \pm 0,03 mm sin encontrar diferencias significativas de tamaño larval entre los tratamientos (Tabla1).

De acuerdo a los resultados de eclosión obtenidos en relación a cada una de las técnicas, se concluye que los métodos de la enzima proteasa, el jugo de piña y el ácido tánico pueden recomendarse para desgomar los huevos de $S$. annulatus.

\section{Agradecimientos}

Los autores agradecen la asistencia técnica del Biol. Acuac. Armando Ibarra y de los señores Manuel Cruz y Juan Huerta y a la alumna Veici Yadira Zepeda y a la Sra. Valerie Williams por la traducción del resumen. Este trabajo fue financiado por el Fondo Mixto Sinaloa (FOMIX) proyecto Sin-2007-C01-71342 dirigido por Luz Estela Rodríguez I.

\section{Literatura citada}

Abdo-de la Parra MI \& NJ Duncan. 2002. Avances en cultivo experimental de botete diana (Sphoeroides annulatus). Panorama Acuícola 7(2): 42-43.
Al Hazzaa R \& A Hussein. 2003. Stickiness elimination of himri barbel (Barbus lutes, Heckel) eggs. Turkish Journal of Fisheries and Aquatic Sciences 3: 47-50.

Álvarez-Lajonchère LS \& O Hernández-Molejón. 2001. Producción de juveniles de peces estuarinos para un centro en América Latina y en el Caribe: diseño, operación y tecnologías, 424 pp. The World Aquaculture Society, Baton Rouge.

Appelbaum S \& R Riehl. 1997. Scanning electron microscopic observations of the chemo and mechanoreceptors of carp larvae (Cyprinus carpio) and their relationship to early behaviour. Aquatic Living Resources 10: 1-12.

Aydin R \& K Köprücü. 2005. Acute toxicity of diazinon on the common carp (Cyprinus carpio L.) embryos and larvae. Pesticide Biochemistry and Physiology 82: 220-225.

Bussing WA. 1995. Tetraodontidae: 1629-1637. En: Fischer W, F Krupp, W Schneider, C Sommer, KE Carpenter \& VH Niem (eds). Guía FAO para la identificación de especies para los fines de la pesca. Pacífico Centro-Oriental. Vol. III. Vertebrados-Parte 2: 1201-1813. FAO, Roma.

Carral JM, JD Celada, M Sáenz-Royuela, R Rodríguez, A Aguilera \& P Melendre. 2006. Effects of four egg desticking procedures on hatching rate and further survival and growth of larvae in the tench (Tinca tinca L.). Aquaculture 237: 632-636.

Chávez-Sánchez MC, LS Álvarez-Lajonchère, MI Abdo-de la Parra \& N García-Aguilar. 2008. Advances in the culture of the Mexican bullseye puffer fish Sphoeroides annulatus, Jenyns (1842). Aquaculture Research 39: 718730 
Demska-Zakés K, Z Zakés \& J Roszuk. 2005. The use of tannic acid to remove adhesiveness from pikeperch, Sander lucioperca, eggs. Aquaculture 236: 1458-1464.

Fujita S \& Y Honma. 1991. Induction of ovarian maturation and development of eggs, larvae and juvenile of the puffer, Takifugu exascurus, reared in the laboratory. Japanese Journal of Ichthyology 38: 211-218.

García-Ortega A. 2009. Nutrition and feeding research in the spotted rose snapper (Lutjanus guttatus) and bullseye puffer (Sphoeroides annulatus), new species for marine aquaculture. Fish Physiology and Biochemistry 35(1): 6980.

Gela D, O Linhart, M Flajšhans \& M Rodina. 2003. Egg incubation time and hatching success in tench Tinca tinca (L.) related to the procedure of egg stickiness elimination. Journal of Applied Ichthyology 19: 132-133.

Kikuchi K, N Iwata, T Kawabata \& T Yanagawa. 2006. Effect of feeding frequency, water temperature, and stocking density on the growth of tiger pufferer Takifugu rubripes. Journal of the World Aquaculture Society 37: 12-20.

Komar C, JF Turnbull, A Roque, E Fajer \& NJ Duncan. 2004. Effect of water treatment and aeration on the percentage hatch of demersal, adhesive eggs of the bullseye puffer (Sphoeroides annulatus). Aquaculture 229: 147-158.

Köprücü K \& R Aydin. 2004. The toxic effects of pyrethroid deltamethrin on the common carp (Cyprinus carpio L.) embryos and larvae. Pesticide Biochemistry and Physiology 80: 47-53.

Kowtal GV, WH Clark Jr \& GN Cherr. 1986. Elimination of adhesiveness in eggs from the white sturgeon, Acipenser transmontanus: chemical treatment of fertilized eggs. Aquaculture 55: 139-143.

Linhart O, D Gela, M Flajšhans, P Duda, M Rodina \& V Novák. 2000. Alcalase enzyme treatment for elimination of egg stickiness in tench, Tinca tinca L. Aquaculture 191: 303-308.

Linhart O, D Gela, M Flajšhans \& M Rodina. 2003a. Proteolytic enzyme treatment: an improved method for elimination of egg stickiness in tench, Tinca tinca L., in aquaculture. Journal of Applied Ichthyology 19: 134-137.
Linhart O, M Rodina, D Gela, M Kocour \& M Rodriguez. 2003b. Improvement of common carp artificial reproduction using enzyme for elimination of egg stickiness. Aquatic Living Resources 16: 450-456.

Linhart O, D Gela, M Rodina \& M Kocour. 2004. Optimization of artificial propagation in European catfish, Silurus glanis L. Aquaculture 235: 619-632.

Linhart O, M Rodina M Kocour \& D Gela. 2006. Insemination, fertilization and management in tench, Tinca tinca (L.). Aquaculture 14: 61-73.

Martínez-Palacios CA, MC Chávez-Sánchez, GS Papp, MI Abdo-de la Parra \& LG Ross. 2002. Observations on spawning early development and growth of the puffer fish Sphoeroides annulatus (Jenyns, 1843). Journal of Aquaculture in the Tropics 17: 59-66.

Martínez-Rodríguez IE, B González-Rodríguez, I Abdo-de la Parra \& N Duncan. 2003. Temperature, salinity affect egg survival and hatching rate in bullseye puffer. Global Aquaculture Advocate 6(3): 28-29.

Rodríguez-Gutiérrez M \& G Garza-Mouriño. 1985. Técnicas para la reproducción inducida de Cyprinus carpio specularis, 10 pp. División de Ciencias Biológicas y de la Salud, Universidad Autónoma Metropolitana, Xochimilco.

Sikkel PC. 1990. Social organization and spawning in the Atlantic sharpnose puffer, Canthigaster rostrata. Environmental Biology of Fishes 27: 243-254.

Thai BT \& TG Ngo. 2004. Use of pineapple juice for elimination of egg stickiness of common carp (Cyprinus carpio L.). Asian Fisheries Science 17: 159-162.

Woynarovich E. 1983. Artificial propagation of Clarias lazera at the Fish Culture Centre Hatchery, Bangui- Landjia. A report prepared for the Hatchery Production and Research Centre Project. FAO GCP/CAF/007/NET- Field Document 1: 1-7.

Zohar Y, G Pagelson, Y Gothilf, WW Dickhoff, P Swanson, S Duguay, W Gombotz, J Kost \& R Langer. 1990. Controlled release of gonadotropin releasing hormones for the manipulation of spawning in farmed fish. Proceedings of the International Symposium on Controlled Release of Bioactive Material 17: 51-52. 